\title{
COMPENSATION OF SPRINGBACK IN LARGE SHEET METAL FORMING
}

\author{
Tomáš PaČÁK*, FrantišeK TatíčEK, Michal Valeš \\ Czech Technical University in Prague, Faculty of Mechanical Engineering, Department of Manufacturing \\ Technology, Technická 1902/4, 16607 Prague, Czech Republic \\ * corresponding author: tomas.pacak@fs.cvut.cz
}

\begin{abstract}
A precise production of sheet metal parts has always been a main goal in press shops. Highest quality demands are required especially in automotive production. Unfortunately, even today, the production is not optimal due to an ineffective approach to the springback compensation. Springback results in geometrical shape inaccuracies of the obtained product. Based on the current approach, excessive time and financial costs emerge due to corrections on the press tools. However, these corrections do not always lead to a better accuracy of the stampings. The main objective of the research is to design a modified solution of the current approach. The modified solution is designed as a methodology with a focus on the analysis and compensation of the springback with a help of a numerical simulation. To achieve the main goal, smaller sub-goals are employed. These sub-goals, or rather experiments, mainly focus on parameters, which, more or less, influence the springback phenomenon. The designed methodology is verified with real car body parts and is carried out with a help of the department of the press tools design in ŠKODA AUTO, a.s. There, the methodology is used for improving the accuracy of the stamping process of the car body parts and for improving the quality of the final product.
\end{abstract}

KeYWORDS: Springback, sheet metal forming, compensation, numerical simulation, AutoForm.

\section{INTRODUCTION}

At the final stage of forming, as soon as the load is removed and stamping tools released, the product springs out. This is caused by internal stresses (elastic deformation). This shift in the material results into change of geometry, this phenomenon is called springback. Springback is a significant problem in the process of sheet metal forming, since it results in geometrical shape inaccuracies of the final product. In order to compensate springback, it is important to carefully consider all factors prior to the stamping process, otherwise a reject product occurs. In most cases, springback is solved in post-production stage of the tools production. That includes intervention into the tools geometry in the form of corrections. This leads to an increase in the costs of the whole project. Ideally, springback should be solved in the pre-production stage of the project. In this stage, various numerical simulations and special computational modules can be used. Tools design and construction are the most time consuming steps in new car body developing process. Therefore, it is very important to find an effective and reliable methodology for a springback prediction and its compensation. [1]

Over the last decades, many researches have investigated the springback phenomenon in the process of sheet metal forming. In particular Yoshida and Uemori have improved the model of large strain plasticity. With the help of this model, it is possible to predict the springback in sheet metal forming more precisely. Nowadays, numerical methods for the process simula- tion and evaluation are commonly used. Software like AutoForm, PAM-STAMP, DynaForm, LS Dyna or others are powerful tools in the pre-production phase. The problem is in the accuracy of these methods, which is still far from exact. If the experimental trial and error process is replaced by a reliable numerical procedure, the pre-production time and costs can be decreased significantly. 2-4]

\section{DESCRIPTION OF THE SPRINGBACK BEHAVIOR}

The dimensional instability caused by springback is highly influenced by the material and process variables. In terms of material variables, it helps to use a material with greater thickness of the sheet, lower material strength and higher Young's modulus. For example, HSS, AHSS and aluminium alloys are predisposed to have a poor dimensional accuracy in comparison with low carbon steels. Each stamping process is unique, due to the variation of each product. A small change in the product geometry can influence the resulting springback significantly. Same applies even for the employed press. Where the inaccuracy of a part differs on a main and replacement press.

Springback can be divided into categories accordingly to the changes in the shape and action of forces. There are a few major categories, e.g. angle deviation, sidewall curl, twist, distortion and global shape change. In the process of pure bending, only one springback type at a time takes place. When we look 
at the more complex deep drawing process, more types of springback take place simultaneously. During this process, various types of the springback influence each other, which makes the description of the problem even more complicated. The springback behaviour can be described with the help of many approaches. Each approach uses different theory for the description and results into differences in the final results. Various limitations of the process conditions cause differences in the final results. One of the less accurate approaches (Rigid - ideally plastic approach) is listed below (eq. 1 and 3). This approach can be applied only on simple bending processes. More accurate approach (Elastic - plastic approach), which can be closely compared to FEM methods, is described with help of eq. 3 , description for the pure bending. [5, 7 ]

Rigid - ideally plastic approach - ideally plastic material without elastic strain and without strain hardening [6]

$$
\begin{gathered}
\frac{M}{w}=2 \int_{0}^{t / 2} \sigma_{0}^{\prime} z \mathrm{~d} z=\frac{\sigma_{0}^{\prime} t^{2}}{4} \\
\frac{R}{r}=1-\frac{3 \sigma_{0}^{\prime} R}{E^{\prime} t}
\end{gathered}
$$

Elastic - plastic approach - elastoplastic relationship in bending process, elastic - plastic power hardening [6]

$$
\begin{aligned}
\frac{\Delta M}{w}=\frac{2 K}{R^{n}(n+2)} & \left(\frac{t}{2}-z^{*}\right)^{n+2}+ \\
& +\frac{2 K z^{*}}{R^{n}(n+1)}\left(\frac{t}{2}-z^{*}\right)^{n+1}
\end{aligned}
$$

For monitoring and analysis of the stamping process, numerical simulations are very useful. One of the commonly used numerical simulation software is AutoForm. AutoForm uses static implicit time integration scheme. In every time step, starting from the previous one, the mesh is regenerated using local refinement. This solving process is iterated until the estimated error is between bounds of the interval of the requiring precision. If the time step between a new iteration is not too large, the time of solving process is usually very small. Even with the help of numerical simulation, it is still very complicated to predict the springback behaviour accurately. Especially in automotive production, where car body parts have a very complex shape, due to the design and body stiffness. 6

Mechanical properties also play a significant role in the springback phenomenon. The dependency of the mechanical properties is, for example, mentioned in the articles [8] or [9]. During our own experiment of a simple bending over a radius, it has been verified that higher the strength of steel material, greater the springback. For example, in the case of $90^{\circ}$ bending of $1.0 \mathrm{~mm}$ sheet over the radius $\mathrm{R} 10$, material
HX340 LAD $\left(R_{m}=441 \mathrm{MPa}\right)$ showed $6.8^{\circ}$ springback, HX220 BD $\left(R_{m}=352 \dot{\mathrm{MPa}}\right) 6.1^{\circ}$ and DX54D $\left(R_{m}=168 \mathrm{MPa}\right) 5.0^{\circ}$. This theory does not contribute to the current trend in the automotive. With more pressure on the lightweight car bodies, high strength steels and smaller thicknesses are applied. That results in even more issues with the total inaccuracy of the shape.

\section{Methodology of the SPRINGBACK ANALYSIS AND COMPENSATION}

In order to resolve the springback phenomenon properly, stable and accurate analysis of the springback must be carried out. With use of the numerical simulations, possibilities in the springback analysis are enormous. The use of the numerical simulations for the springback analysis has mostly advantages, however, some drawbacks are also present (besides the persisting lower accuracy). The advantages are mainly in the analysis itself, where all kinds of comparisons can be used. Such as an evaluation in various directions, comparison with the reference geometry and more.

On the contrary, when it comes to the springback analysis, conditions of the analysis play a great role in the final accuracy. For example, a part can be evaluated with no gravity taken into account (Free Springback), with gravity (Constrained Springback) or with gravity and with progressive clamping (Real Measurement). Results with an application of each analysis always vary. Furthermore, settings of the initial numerical simulation also influence the final results of the springback analysis. For instance, settings of finite element method (type and size of elements, nodes, number of iterations, etc.) and process parameters (pressure of binder, drawbead type, trimming with or without tools, pressure and velocity dependency, etc.). Possibilities in the combination of various conditions and settings in the numerical simulation are very comprehensive. The aim is to reach accurate results that can be achieved through a unified methodology. Such methodology is shown in the Fig. 1. This methodology also represents the modified approach to the springback phenomenon. [5]

At the moment, when analysis and compensation of springback is expected, numerical simulation must be carefully designed from the very beginning. As mentioned, the overall accuracy of the springback analysis is highly influenced by the process variables and initial settings of the numerical simulation. In the purpose of creating a unified methodology, a checklist for the numerical simulation had to be designed foremost. Optimal options for the settings of numerical simulations were obtained through various tests. Below are listed only the key steps and major parts of the checklist: [7] 


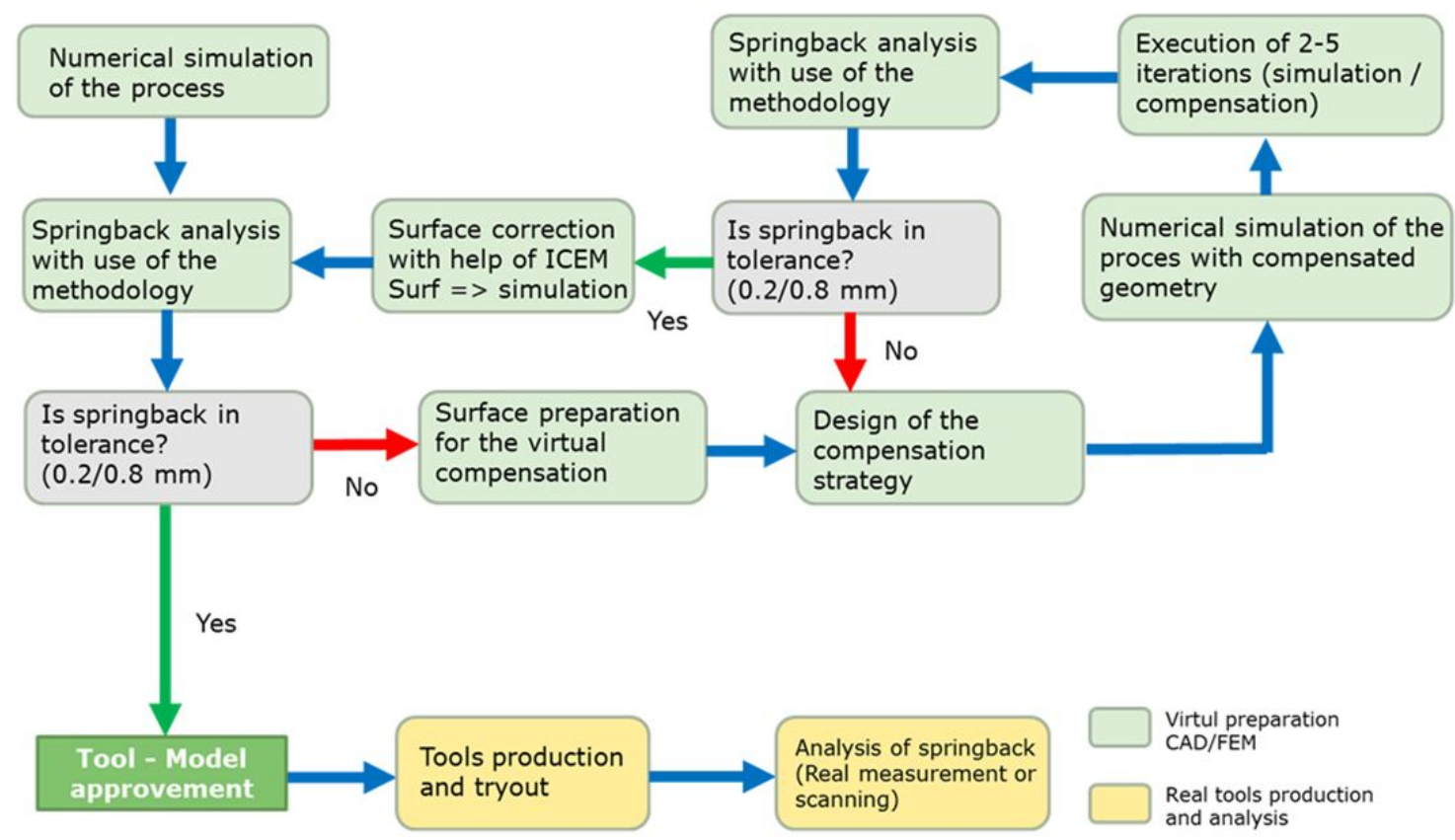

FIGURE 1. Schema of the modified solution of the approach to the springback phenomenon with greater focus on the pre-production, virtual part of the project.

- Settings of imported geometry - meshing tolerance, stitching distance, max side length, master element size, max element angle, etc.

- FEM calculation - use of elastic plastic shell elements instead of membrane cells.

- Stamping process - use of the 3D or adaptive drawbeads, application of pressure and velocity dependency, use of more complex description of friction, etc.

- Process in numerical simulation corresponds with the productive press line.

- Trimming and cutting operations with complete tool geometry.

- Radius and thickness ratio $R / t<2$.

- Use of pilots due to the centring of blank position.

The most important source of inaccuracy of the springback in numerical simulations is the analysis itself. Only with a virtual analysis, which is identical with the real measurement, accurate results can be achieved. Compact methodology for the springback analysis is shown in the figure 2 .

When it comes to the springback compensation, two approaches can be applied. First and current approach focuses on the manual geometry compensation with the CAD modelling. This approach, oftentimes spring-forward method, requires a lot of experience in the field of forming. Also this approach is time consuming due to the manual surface modelling. The more effective approach is the use of a special computational modules, e.g. AutoForm Compensator or PAMSTAMP Die Compensation module. These modules focus on the geometry correction after the springback analysis from the previous iteration. The principle is similar to the manual correction - to create a compensated tool geometry which will help to achieve a better dimensional accuracy. However, to get such results from the compensation, a very consistent fundamental simulation with appropriate compensation strategy must be used. 6]

Results from the compensation can differ because of various possibilities in the approach to the compensation. Compensation strategy focuses on the:

- Selection of the stamping operation for the following compensation.

- Selection of the tools geometry (fixed, compensated or transitional).

- Definition of a compensation ratio.

Above, a compensation ratio was mentioned. With the help of the ratio, the intensity of the compensation can be influenced. With a smaller ratio, tool's geometry is compensated only slightly. This results into smaller changes in the tools geometry and thus improved feasibility in the tools try-out. On the contrary, a higher ratio results in a better elimination of springback. Based on the experiments, an optimal ratio was found to be in the interval between $\mathbf{0 . 2}$ to 0.4. When a higher ratio is applied, the continuity of the tool's geometry is fragmented. This will lead to a poor tool's feasibility. [7]

\section{VERIFICATION OF THE SPRINGBACK COMPENSATION METHODOLOGY}

The main motivation behind the verification was to discover if the modified approach is feasible and bene- 


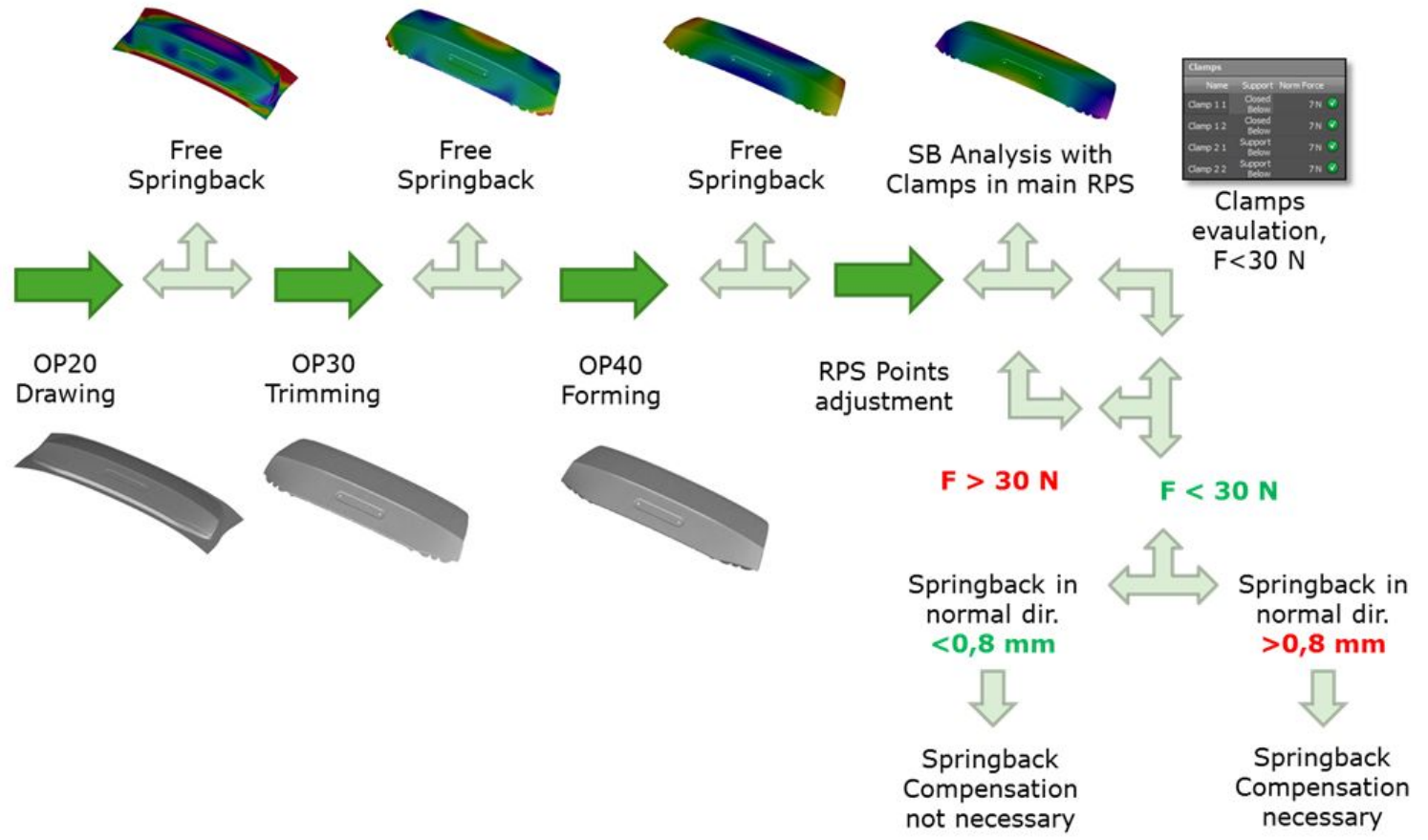

FIGURE 2. Schema of the methodology of the virtual springback analysis [5].

ficial for future projects. The described methodology has been carried out on car body parts with a various geometry complexity. Namely:

- SEAT Ateca - outer bottom panel from fifth doors $\mathrm{DC} 06+\mathrm{ZE}$

- SEAT Ateca - inner fifth door panel DX57D

- ŠKODA Superb - fender HX220 BD

The methodology was verified through a process of a numerical simulation, springback analysis and comparison with the reference geometry. Finally, a compensation according to the methodology has been carried out. Car body parts with a less complex geometry underwent the verification relatively well. This applies to the outer and inner panel of fifth doors from SEAT Ateca. On these parts, the verification of springback analysis was successful (maximum deviation from real-life scan circa $0.5 \mathrm{~mm}$ ). Also the springback compensation was successful, where accuracy of the shape was improved. Maximum dimensional inaccuracy was circa $0.5 \mathrm{~mm}$ on the outer fifth door panel and circa $0.6 \mathrm{~mm}$ on the inner fifth door panel.

As mentioned, each compensation strategy results into different results. Specifically, on the outer fifth door panel, total of 5 strategies have been applied. Results from each strategy and its iterations are shown in Fig. 3. The best results have been achieved with the $1^{\text {st }}$ strategy where tools are compensated in every stamping operation. Fig. 4 displays the springback analysis of the initial numerical simulation and analysis after the final iteration of the springback compensation ( $1^{\text {st }}$ strategy).

In terms of more complex geometry and forming processes, the methodology was not successful. In the case of the fender from ŠKODA Superb, both the springback analysis and compensation did not achieve satisfying results. Thanks to the compensation, springback has been improved from the initial maximum value $7.5 \mathrm{~mm}$ to the final $3 \mathrm{~mm}$. Even though dimensional accuracy has been improved significantly, it still did not meet the requirements $(0.8 \mathrm{~mm})$. A total of 4 strategies has been applied and resulted with similar or worse results.

Based on the application of the designed methodology and its verification, car body parts have been distributed into categories of complexity (figure 5). Each category describes its complexity from the point of view of a forming process and geometry of the part. Also a status has been added to describe if it is currently possible to successfully use the methodology on the parts from each category.

\section{Comparison of the Results}

Evaluations presented in this paper show a similar tendency as the current literature. This paper corresponds with the statement that springback phenomenon can currently be accurately analysed and compensated only on parts with a less complex shape. Through literature, most papers agree that various compensation strategies lead to an improvement in the inaccuracy by up to $70-80 \%$ [10, 12. Similar but slightly better results have been achieved in the presented experiment. With the designed strategy, the improvement of the inaccuracy achieved up to 80-90\% in the case of SEAT Ateca fifth door parts.

Some researches rate the accuracy of springback phenomenon even more critically. Since the calculation of the springback prediction is not able to reach 


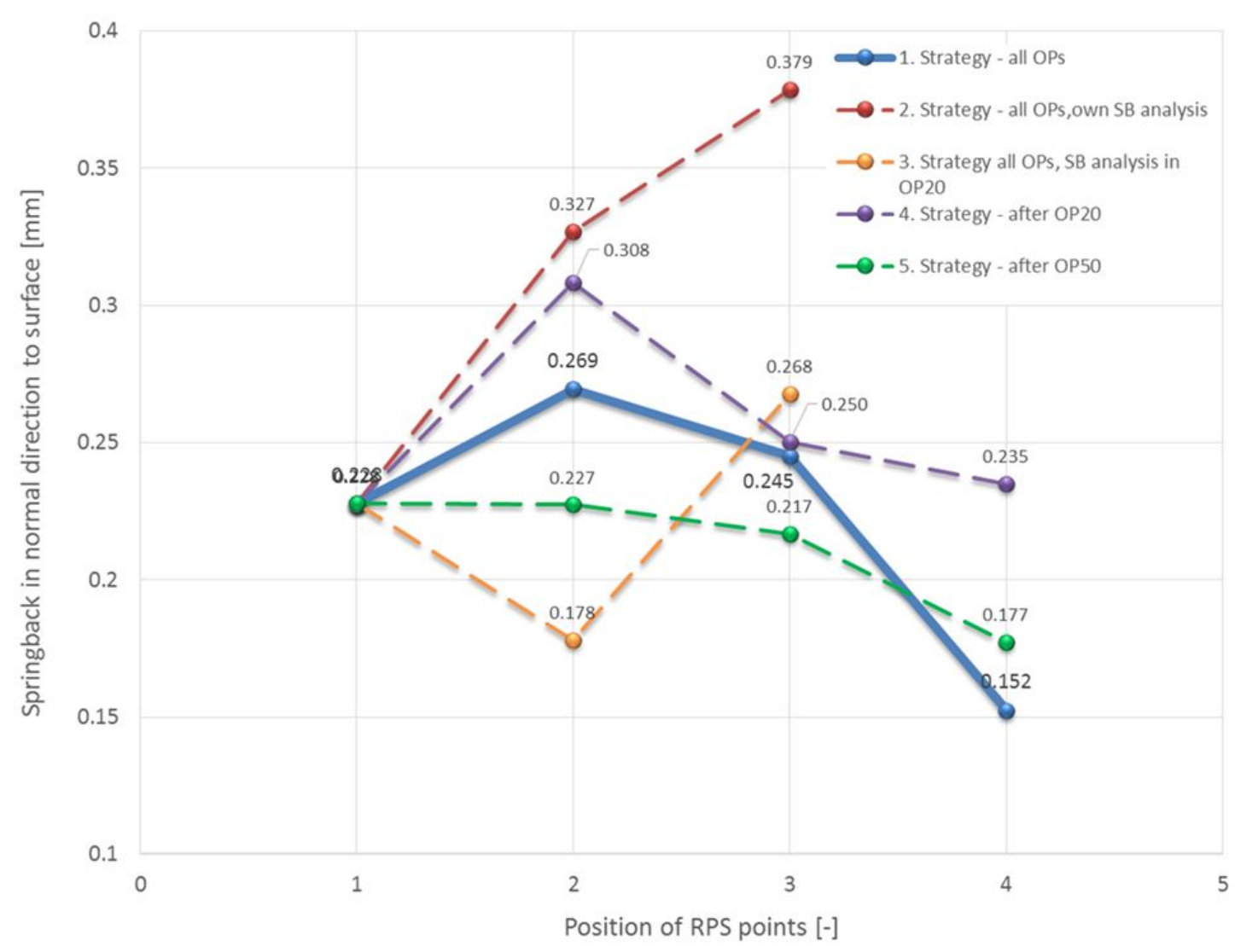

FIGURE 3. Graphical representation of springback results after application of various compensation strategies (each number represents a magnitude of the springback defined by the springback coefficient $\mathrm{Si}$ ) [7].

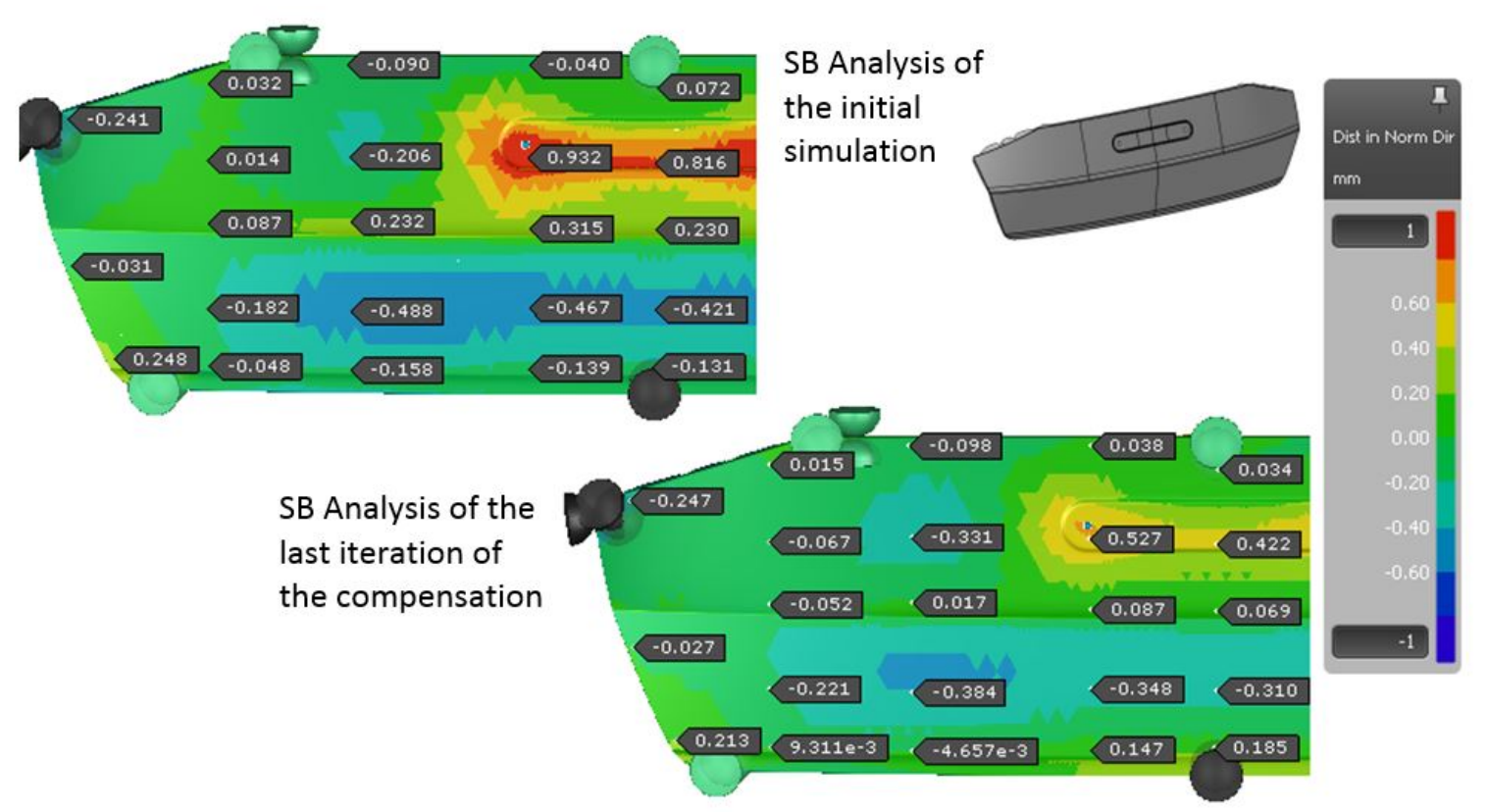

FiguRE 4. Results from compensation strategy no. 1 - comparison between initial springback analysis and last iteration of the compensation (SB compensation consist of iterations in total) [7]. 


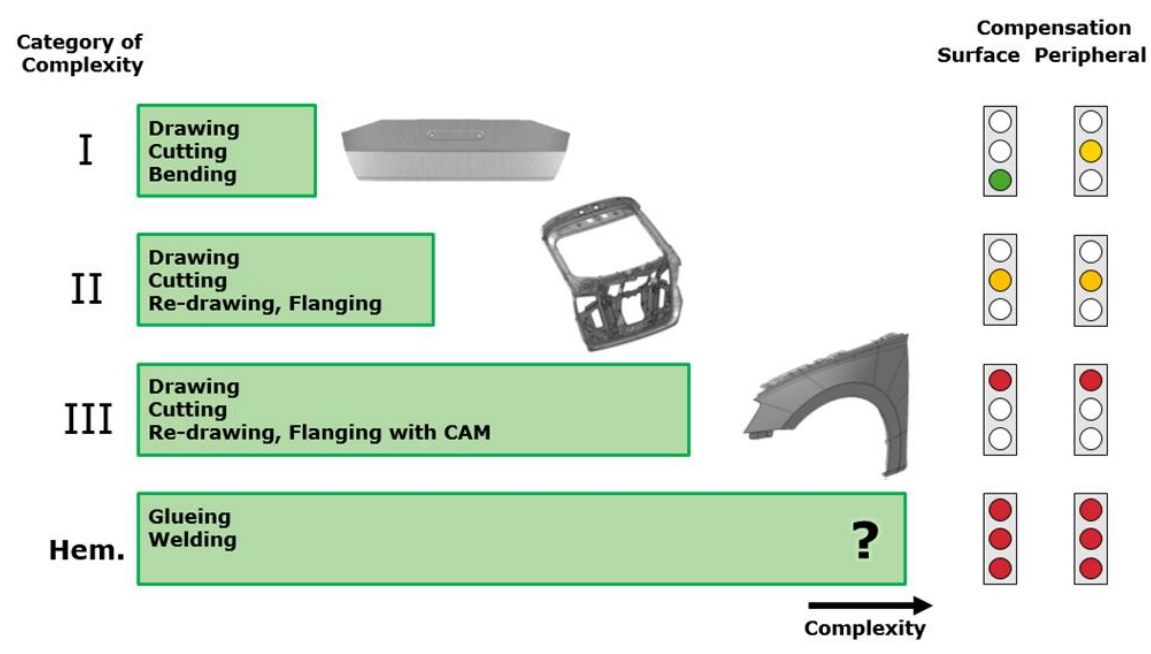

Figure 5. Categories of car body parts based on the complexity of the forming process and of the geometry. Each category is evaluated from the point of view of the springback prediction and its compensation [7.

$100 \%$ of the accuracy, the following compensation should be even more inexact (the paper mentions overall accuracy $56 \%$ even with the remodelling the tools geometry) 13. Similar trend has been achieved with the experiment on the ŠKODA Octavia fender, where the accuracy has not been acceptable.

\section{Conclusion}

Existence of the springback in stamping processes is very common. In terms of bending operations, springback has already been described by many authors. The description of the springback behaviour is more complicated in the case of consecutive stamping operations in the press line, In the process of forming, springback is influenced by a number of process variables. Some of the conditions influence springback extensively (material properties, material's thickness, friction, plastic strain, etc.). The initial settings of numerical simulation also have an additional impact. A major condition is that the numerical simulation has to be carried out under the same conditions as in the real stamping process.

The research is based on the modified solution in the virtual approach to the springback analysis and compensation. An integral part of the modified solution is the methodology. The methodology consists of three main parts: correct settings of virtual forming process (numerical simulation), springback analysis and springback compensation. The methodology has been employed on car body parts with a various geometry and various forming process (Chapter 4). Results from the numerical simulation have been compared to digital scans of produced parts (based on a real stamping process in a press shop). The verification proved that an accurate springback analysis and its compensation is challenging. Figure 5 summarize the possible application on various types of car body parts.

With more complex part geometry and with the virtual solution, it is difficult to accomplish accurate results. In addition, accurate virtual solving of compound parts with hemming is nearly impossible. The reason is the description of a springback when assembly parts are hemmed together. Another problematic step after the virtual compensation is a curvature analysis of the compensated geometry. The curvature of the surface is mostly uneven and wavy due to the local geometry adjustment. It is complicated to mill such surface and later to fit bottom and upper tools together in the try-out press. Therefore, after the local springback compensation, surface has to be smoothened with a special software.

The modified virtual solution is beneficial for car body parts but with a condition of application on less complex geometry, e.g. fifth door outer panels, inner and outer doors or inner and outer bonnets. The same applies on the smaller structural car body parts. Here, the designed solution can highly improve the final accuracy and decrease the time and financial costs during the pre- and post-production [7].

\section{ACKNOWLEDGEMENTS}

The research was financed by SGS16/217/OHK2/3T/12. Sustainable Research and Development in the Field of Manufacturing Technology.

\section{REFERENCES}

[1] A. Soualem. A detailed experimental study and evaluation of springback under stretch bending process. International Journal of Aerospace and Mechanical Engineering 8(6): 1128 - 1131, 2014. DOI:10.5281/zenodo.1093219.

[2] A. H. Alghtani, P. C. Brooks, D. C. Barton, V. V. Toropov. Springback analysis and optimization in sheet metal forming. In 9th European LS-Dyna Conference 2013. 2013.

[3] Department of ferrous metallurgy. Springback of high strength automotive steels. http: //www.iehk.rwth-aachen. de/index .php?id=503\&L=2 
[4] T. Yoshida, E. Isogai, S. Yonemura, et al. Material modeling for accuracy improvement of thespringback prediction of high-strength steel sheets. technical report 102, Nippon Steel, 2013.

[5] T. Pačák, et al. Methodology of the springback compensation in sheet metal stamping processes. In METAL 2017 Conference Proceedings, pp. 502-507. Brno, 2018.

[6] ASM Handbook. Metalworking: Sheet Forming, vol. 14b. ASM International, Ohio, 2006.

[7] T. Pačák, et al. Compensation of the springback behavior in large sheet metal stamping. In Book of Proceeding from Conference Technological Forum 2018. Jaroměr̆, 2018.

[8] S. Benson. Bending Basics: The hows and whys of springback and springforward, 2014.

https://www. thefabricator. com/thefabricator/

article/bending/bending-basics-the-hows-

and-whys-of-springback-and-springforward
[9] D.-K. Leu, Z.-W. Zhuang. Springback prediction of the vee bending process for high-strength steel sheets. Journal of Mechanical Science and Technology 30(3):1077 - 1084, 2016. DOI:10.1007/s12206-016-0212-8

[10] J. Weiher, B. Rietman, K. Kose, et al. Controlling springback with compensation strategies. AIP Conference Proceedings 712(1):1011 - 1015, 2004. DOI:10.1063/1.1766660

[11] W. A. Siswanto, A. D. Anggono, B. Omar, K. Jusoff. An alternate method to springback compensation for sheet metal forming. The Scientific World Journal 2014:1 - 13, 2014. DOI:10.1155/2014/301271.

[12] S. Xu, K. Zhao, T. Lanker, et al. Springback prediction, compensation and correlation for automotive stamping. AIP Conference Proceedings 778(1):345 352, 2005. DOI:10.1063/1.2011244.

[13] R. Lingbeek. Aspects of a design tool for springback compensation. Master's thesis, University of Twente / INPRO, 2003. 